\title{
ON THREE PROBLEMS CONCERNING NIL-RINGS
}

\section{JAKOB LEVITZKI}

1. Introduction. In the present note three problems concerning nil-rings are proposed and certain relations linking these problems to one another are discussed.

First problem. The sum of all two-sided ${ }^{1}$ nil-ideals of a ring $S$ has been defined by $\mathrm{G}$. Koethe $[2, \S 3]^{2}$ as the radical of $S$, provided that this sum contains also all one-sided nil-ideals of $S$. We shall henceforth refer to this radical as the $K$-radical ${ }^{3}$ of $S$. It is an open question whether or not there are rings in which the $K$-radical does not exist.

Second problem. A ring $T$ is called semi-nilpotent (see [3, §2]) if each finite set of elements in $T$ generates a nilpotent ring. A ring which is not semi-nilpotent is called semi-regular. Each semi-nilpotent ring is evidently a nil-ring. It is an open question whether or not there exist semi-regular nil-rings. As may easily be seen, this problem is equivalent to the question whether or not there exist semi-regular nil-rings which are generated by a finite set of elements.

Third problem. A nil-ideal $P$ of a ring $S$ has been termed by R. Baer $[1, \S 1]$ a radical ideal if the quotient-ring $S / P$ does not contain nilpotent ideals other than zero. The sum $U(S)$ and the crosscut $L(S)$ of all radical ideals of a ring $S$ are again radical ideals which are called the upper radical and the lower radical respectively (see Baer $[1, \S 1]$ ). As indicated by Baer ideals may exist between $U(S)$ and $L(S)$ which are not radical ideals. $R$. Baer has also constructed an interesting example which illustrates this possibility. Our results in the present note show that this phenomenon can not be considered as an exception to the rule but on the contrary rather as the rule itself, and thus the following problem presents itself: Are there or are there not rings $S$ in which $U(S) \supset L(S)$ and in which furthermore each ideal which lies between $U(S)$ and $L(S)$ is also a radical ideal?

In the present note the following results are obtained: Suppose that $S$ is a ring in which the $K$-radical does not exist, then $S$ contains an infinite number of right ideals as well as of left ideals which are semi-regular nil-rings (see Theorem 4 in $\S 3$ ). Suppose that $S$ is a ring with a semi-regular upper radical $U(S)$, then $S$ contains a subring $S^{\prime}$ so that $U(S) \supseteq S^{\prime}=U\left(S^{\prime}\right) \supset L\left(S^{\prime}\right)$ and so that $S^{\prime}$ contains an infinite

Received by the editors April 3, 1945.

1 We shall write henceforth in short ideals instead of two-sided ideals.

2 Numbers in brackets refer to the Bibliography at the end of the paper.

${ }^{3}$ For the sake of convenience we shall reserve in this paper the term radical for the sum of all semi-nilpotent ideals of the ring (see $[3, \S 2]$ ). 
number of ideals between $U\left(S^{\prime}\right)$ and $L\left(S^{\prime}\right)$ which are not radical ideals (see Theorem 5 in $\S 3$ ). If $S$ is a ring with a semi-nilpotent upper radical $U(S)$, and if $U(S) \supset L(S)$, then $S$ contains an infinite number of ideals between $U(S)$ and $L(S)$ which are not radical ideals (see Theorem 6 in $\$ 3$ ).

Remarks. From Theorem 4 it follows that if the first problem is answered in the affirmative then this will apply also to the second problem. From Theorem 5 it follows that if the second problem is answered in the negative then this will also apply to the third problem. The above-mentioned example of $\mathrm{R}$. Baer is of a nil-ring $U$ of which it can be easily proved that it is semi-nilpotent. In fact, the proof for the semi-nilpotency of $U$ is implicitly contained in Baer's proof for the nillity of $U$ (see $[1, \S 2]$ ). Thus our Theorem 6 explains the phenomenon which was described by Baer in his example. This theorem in conjunction with Theorem $\mathbf{5}$ seems to justify the conjecture that the answer to the third problem is in the negative.

Notations. If the ring $T$ is generated by the finite set of elements $t_{1}, t_{2}, \cdots, t_{n}$, then we write $T=\left\{t_{1}, t_{2}, \cdots, t_{n}\right\}$ or $T=\{\cdots$, $\left.t_{i}, \cdots\right\}$. The sum ${ }^{4}$ of a finite number of right ideals (left ideals) $A_{1}, A_{2}, \cdots, A_{n}$ will be denoted by $A_{1}+A_{2}+\cdots+A_{n}=\sum_{i=1}^{n} A_{i}$.

The ideal, the right ideal and the left ideal in the ring $S$ which are generated by a finite set of elements $a_{1}, a_{2}, \cdots, a_{n}$ will be denoted by $\left(a_{1}, a_{2}, \cdots, a_{n}\right),\left(a_{1}, a_{2}, \cdots, a_{n}\right)_{r}$ and $\left(a_{1}, a_{2}, \cdots, a_{n}\right)_{l}$ respectively. If $A=\left(a_{1}, a_{2}, \cdots, a_{n}\right)_{r}$, then $A \supseteq \sum_{i=1}^{n} a_{i} S$. In case $A=\sum_{i=1}^{n} a_{i} S$ we say that $A$ possesses a proper right basis. Similarly a proper left basis is defined.

If $A=\left(a_{1}, a_{2}, \cdots, a_{n}\right)$, then $A \supseteq \sum_{i=1}^{n} S \cdot a_{i} S$. In case $A=\sum_{i=1}^{n} S a_{i} S$ we say that $A$ possesses the proper basis $a_{1}, a_{2}, \cdots, a_{n}$.

If the ring $S$ has an identity element, then all bases are of course proper bases.

2. On nil-ideals with proper bases. In this section we shall derive certain properties of nil-ideals with proper bases which will be used in $\$ 3$ for the proof of the assertions made in the introduction.

THEOREM 1. If $R$ is a nonzero nil-ideal with a proper right basis in a ring $S$, then $R^{2} \subset R$.

Proof. By assumption we may put $R=\sum_{i=1}^{n} a_{i} S$, where $a_{i} \in R$, $i=1, \cdots, n$. Since further $S a_{i} S \subseteq R$, we have $R^{2}=\sum_{i, k=1}^{n} a_{i} S a_{k} S$ $\subseteq \sum_{i=1}^{n} a_{i} R \subseteq R^{2}$, and hence $R^{2}=\sum_{i=1}^{n} a_{i} R$. Now suppose that $R^{2} \nsubseteq R$, then by $R^{2} \subseteq R$ we would have $R^{2}=R$, that is,

\footnotetext{
* We shall not use direct sums in this paper.
} 


$$
R=\sum_{i=1}^{n} a_{i} R, \quad a_{i} \in R, i=1, \cdots, n .
$$

In case $n=1$ we obtain $R=a_{1} R$, which by successive left multiplication yields $R=a_{1}^{k} R$ for each $k$, that is, $a_{1}^{\boldsymbol{k}} \neq 0$ for each $k$, which is a contradiction to the nillity of $R$. Now suppose that $n \geqq 2$ and define $m$ so that

$$
R=\sum_{i=1}^{m} a_{i} R, \quad R \supset \sum_{i=1}^{m-1} a_{i} R, \quad 2 \leqq m \leqq n .
$$

By putting $T=\sum_{i=1}^{m-1} a_{i} R$ we then have

$$
R=T+a_{m} R,
$$$$
a_{m} \neq 0 .
$$

By (3) it follows that $a_{m}$ can be represented in the form

$$
a_{m}=b_{1}+a_{m} c, \quad b_{1} \in T, c \in R .
$$

Now suppose that for a certain $r$ it has been proved that $a_{m}=b_{r}+a_{m} c^{r}$, $b_{r} \in T$, then by (4) this would yield $a_{m}=b_{r}+\left(b_{1}+a_{m} c\right) c^{r}=b_{r+1}+a_{m} c^{r+1}$, where $b_{r+1}=b_{r}+b_{1} c^{r}$. In view of the fact that $T$ is a right ideal, it follows that $b_{r+1} \in T$, and thus by induction we have proved that for each $s$ the element $a_{m}$ has a representation of the form

$$
a_{m}=b_{s}+a_{m} c^{s}, \quad b_{s} \in T, c \in R .
$$

Now take for $s$ the index of the nilpotent element $c$, then $c^{s}=0$, and we have $a_{m}=b_{s} \in T$, and hence $a_{m} R \subseteq T$, or $R=\sum_{i=1}^{m-1} a_{i} R$, which is a contradiction to (2). Consequently equation (1) is impossible, which implies that $R^{2} \subset R$.

THEOREM 2. If $R$ is a nonzero semi-nilpotent right ideal with a proper right basis in a ring $S$, then $R^{2} \subset R$.

Proof. For a certain integer $n$ we have by assumption $R=\sum_{i=1}^{n} a_{i} S$ with $a_{i} \in R, i=1, \cdots, n$. Now denote by $N$ the $\operatorname{radical}^{3}$ of $S$, then $R \subseteq N$, and $S a_{i} S \subseteq N$ (see [3, §2]). Hence we have $R^{2}=\sum_{k, k=1}^{n} a_{i} S a_{k} S$ $\subseteq \sum_{i=1}^{n} a_{i} N$. Now suppose that $R^{2} \subsetneq R$, then we would have $R^{2}=R$, that is, $R \subseteq \sum_{i=1}^{n} a_{i} N \subseteq R$, or

$$
R=\sum_{i=1}^{n} a_{i} N, \quad a_{i} \in R, i=1, \cdots, n .
$$

From (6) follows 


$$
a_{i}=\sum_{k=1}^{n} a_{k} b_{i k}, \quad b_{i k} \in N, i, k=1, \cdots, n .
$$

By considering the ring $B=\left\{\cdots, b_{i k}, \cdots\right\}$, we have in view of (7) the relation $a_{i} \in \sum_{k=1}^{n} a_{k} B, i=1, \cdots, n$, which by right multiplication yields $a_{i} B \subseteq \sum_{k=1}^{n} a_{k} B^{2}, i=1, \cdots, n$, and hence $a_{i} \in \sum_{k=1}^{n} a_{k} B^{2}$, $i=1, \cdots, n$. Thus by successive right multiplication we obtain for each $m$

$$
a_{i} \in \sum_{k=1}^{n} a_{k} B^{m}, \quad i=1, \cdots, n,
$$

which implies that $B^{m} \neq 0$ for each $m$. But this is a contradiction, since $N$ is semi-nilpotent, $B \subseteq N$, and $B$ is generated by a finite set of elements.

Remark. Each nil-ideal of a ring $S$ is contained in the upper radical $U(S)$ of $S$. Only a slight modification of the proof of Theorem 1 is necessary in order to extend the validity of that theorem to one-sided ideals of $S$ which are contained in $U(S)$. In this generalized form, Theorem 1 would include Theorem 2 as a special case, since each semi-nilpotent one-sided ideal lies in the radical ${ }^{3} N$ of $S$, which in turn is a subset of the upper radical.

THEOREM 3. If $R$ is a nonzero semi-nilpotent ideal with a proper basis in $S$, then $R^{2} \subset R$.

PRoof. For a certain integer $n$ we have by assumption $R=\sum_{i=1}^{n} S a_{i} S, a_{i} \in R, i=1, \cdots, n$. If, again, $N$ denotes the radical ${ }^{3}$ of $S$, then $S a_{i} S \subseteq N$ for each $i$, and hence we have $S a_{i} S S a_{k} S S a_{j} S$ $\subseteq N a_{k} N$ for any triple of indices $i, j, k$. Thus it follows that $R^{3}=\sum_{k, k, j=1}^{n} S a_{i} S S a_{k} S S a_{j} S \subseteq \sum_{k=1}^{n} N a_{k} N$. Now suppose that $R^{2} \llbracket R$, then we would have $R^{2}=R$, and hence also $R^{3}=R$, which by $R=R^{3} \subseteq \sum_{k=1}^{n} N a_{k} N \subseteq R$ implies

$$
R=\sum_{k=1}^{n} N a_{k} N, \quad a_{k} \in R, k=1, \cdots, n .
$$

By (9) it follows that each $a_{k}$ has the form

$$
a_{k}=\sum_{i=1}^{n} b_{k i} a_{i} c_{k i}, \quad b_{k i} \in N, c_{k i} \in N, i, k=1, \cdots, n \text {. }
$$

Putting $B=\left\{\cdots, b_{i k}, \cdots\right\}$ and $C=\left\{\cdots, c_{i k}, \cdots\right\}$ we can write (10) in the form $a_{k} \in \sum_{i=1}^{n} B a_{i} C, k=1, \cdots, n$, which by successive right and left multiplication yields 


$$
a_{k} \in \sum_{i=1}^{n} B^{m} a_{i} C^{m}, \quad k=1, \cdots, n \text {. }
$$

By (11) it follows that $B^{m} \neq 0, C^{m} \neq 0$ for each $m$, which is a contradiction to the semi-nilpotency of $N$.

3. Proof of statements made in the introduction. The proof of the following Theorem 4 follows directly from the author's results in $[3, \S 2]$, while Theorems 5 and 6 are based on our results in $\$ 2$.

THEOREM 4. If $S$ is a ring in which the $K$-radical does not exist, then $S$ contains an infinite number of right ideals as well as of left ideals which are semi-regular nil-rings.

Proof. First note that $S$ contains right nil-ideals as well as left nil-ideals which are not in the upper radical $U(S)$ of $S$. Indeed, by assumption $S$ contains a one-sided nil-ideal $A$ so that $A \subset U(S)$. Now define the element $a$ so that $a \in A$ but $a \notin U(S)$, then the right ideal $(a)_{r}$ and the left ideal $(a)_{l}$ are nil-ideals which are not in $U(S)$. Now denote by $R$ any right nil-ideal so that $R \nsubseteq U(S)$, then also $R^{2} \leftarrow U(S)$, since $U(S)$ is a radical ideal. Consequently $R$ contains an element $a_{1}$ so that $a_{1} R \nsubseteq U(S)$. By $a_{1} R \subseteq R$ and by the nillity of $R$ follows easily $R \supset a_{1} R$. Now put $R_{1}=a_{1} R$; then in view of $R_{1} \varnothing U(S)$ we may repeat with $R_{1}$ the same procedure, and thus by induction obtain an infinite sequence of right nil-ideals $R_{1}, R_{2}, R_{3}, \cdots$, each satisfying the relation $R_{i} \nsubseteq U(S)$. Now each semi-nilpotent right ideal of $S$ is contained in the radical ${ }^{3} N$ of $S$ (see $[3, \S 2]$ ) which is a subset of $U(S)$. This implies that the right ideals of the sequence $R_{1}, R_{2}, \cdots$ are semiregular; since a similar result holds for left ideals, the proof of our theorem is thus completed.

LEMMA 1. If $T$ is a ring with a finite set of generators, then for each positive integer $n$ also $T^{n}$ is a ring with a finite number of generators.

Proof. Write $T=\left\{a_{1}, a_{2}, \cdots, a_{m}\right\}$, and put $b_{i_{1}, i_{2}, \cdots, i_{n+k}}$ $=a_{i_{1}} a_{i_{2}} \cdots a_{i_{n+k}}$, where $0 \leqq k<n$ and $1 \leqq i_{j} \leqq m$ for $j=1, \cdots, n+k$; then, as may easily be seen, $T^{n}=\left\{\cdots, b_{i_{1}, i_{2}}, \cdots, i_{n+k}, \cdots\right\}$, q.e.d.

LEMMA 2. If T is a semi-regular nil-ring with a finite set of generators, then $T^{n} \supset T^{n+1}$ for each positive integer $n$.

Proof. By Lemma 1 we may put $T^{n}=\left\{b_{1}, b_{2}, \cdots, b_{r}\right\}$. Now suppose that $T^{n}=T^{n+1}$, then by successive multiplication follows $T^{n}=T^{n+m}$ for each $m$, and hence by putting $T^{n}=W$ we have $W=W^{k}$ for each $k$. In view of $W=\left\{b_{1}, \cdots, b_{r}\right\}$ it follows easily that 
$W^{2}=\sum_{i=1}^{r} b_{i} W=W$, which is a contradiction to Theorem 1.

Lemma 3. If $P$ and $Q$ are nil-ideals of $a$ ring $S$ and if $P \supseteq Q$, then $P$ is a radical ideal if and only if $P / Q$ is a radical ideal in the ring $S / Q$. In particular, $Q$ is a radical ideal if and only if the zero ideal is a radical ideal in $S / Q$.

Proof. Denote by $A$ a two-sided ideal of $S$ so that $A \supseteq P$, then by the so-called "second law of isomorphisms" (see [4, p. 149]) we have $(A / Q) /(P / Q) \cong A / P$. Our lemma follows from the fact that $A / P$ is nilpotent if and only if $(A / Q) /(P / Q)$ is nilpotent.

LemMa 4. If the lower radical of a ring $S$ is zero and if the upper radical $U(S)$ is semi-regular, then $S$ contains a subring $T$ so that $U(S) \supseteq T=U(T) \supset L(T)$ and $T$ contains an infinite number of ideals between $U(T)$ and $L(T)$ which are not radical ideals.

Proof. By assumption $U(S)$ contains a finite set of elements $a_{1}, a_{2}, \cdots, a_{n}$ so that the ring $T=\left\{a_{1}, a_{2}, \cdots, a_{n}\right\}$ is a semi-regular nil-ring. In view of the semi-regularity of $U(T)$ and the seminilpotency of the lower radical ${ }^{5}$ it follows that $T=U(T) \supset L(T)$, and that also the quotient-ring $W=T / L(T)$ is a semi-regular nil-ring. If now $b_{i}$ denotes the image of $a_{i}$ in the homomorphism $T \sim W$, we may evidently write $W=\left\{b_{1}, b_{2}, \cdots, b_{n}\right\}$. By Lemma 2 we have $W^{m+r} \subset W^{m}$ for any pair of positive integers $m, r$, that is, the nonzero ideal $W^{m} / W^{m+r}$ of the ring $T / W^{m+r}$ is nilpotent. Consequently, $W^{m+r}$ is not a radical ideal of $W$. Now define for each positive integer $r$ the ideal $A_{r}$ of $T$ by the relations $A_{r} \supseteq L(T), A_{r} / L(T) \cong W^{r}$, then $A_{r}$ is uniquely determined, and in view of Lemma 3 none of the ideals of the infinite sequence $A_{2}, A_{3}, \cdots$ is a radical ideal, q.e.d.

LEMMA 5. If the lower radical of a ring $S$ is zero and if $A$ is a nonzero semi-nilpotent ideal of $S$, then $S$ contains an infinite number of ideals which are not radical ideals and which are subsets of $A$.

Proof. Denote by $a_{1}, a_{2}, \cdots, a_{n}$ an arbitrary finite set of nonzero elements of $A$ and consider the nonzero ideal $A_{1}=\left(a_{1}, a_{2}, \cdots, a_{n}\right)$ of $S$ which lies in $A$. As may easily be verified, we then have $A_{1}^{3}=\sum_{i=1}^{n} A_{1} a_{i} A_{1}$. Now suppose that $A_{1}^{3}=A_{1}$, then we would have $A_{1}=\sum_{i=1}^{n} A_{1} a_{i} A_{1} \subseteq \sum_{i=1}^{n} S a_{i} S \subseteq A_{1}$, that is, $A_{1}=\sum_{i=1}^{n} S a_{i} S$, which is a contradiction to Theorem 3 in $\$ 2$. Hence we have $A_{1}^{3} \subset A_{1}$, which implies that the ideal $A_{1} / A_{1}^{3}$ of the ring $S / A_{1}^{3}$ is nilpotent, that is,

\footnotetext{
${ }^{5}$ The lower radical is semi-nilpotent since it is a subset of the radical $N$ (see footnote 2).
} 
$B_{1}=A_{1}^{3}$ is not a radical ideal. By $L(S)=0$ it follows that $B_{1} \supset 0$. Since $0 \subset B_{1} \subset A$, we may repeat with $B_{1}$ the same procedure, and thus (by induction) obtain an infinite sequence of semi-nilpotent ideals $B_{1} \supset B_{2} \supset B_{3} \supset \cdots$ none of which is a radical ideal, q.e.d.

THEOREM 5. If $S$ is a ring with a semi-regular upper radical $U(S)$, then $S$ contains a subring $S^{\prime}$ so that $U(S) \supseteq S^{\prime}=U\left(S^{\prime}\right) \supset L\left(S^{\prime}\right)$ and so that $S^{\prime}$ contains an infinite number of ideals between $U\left(S^{\prime}\right)$ and $L\left(S^{\prime}\right)$ which are not radical ideals.

Proof. By Lemma 3 (see also Baer $[1$, p. 539]) the lower radical of $S^{*}=S / L(S)$ is zero, while the upper radical $U(S) / L(S)=U\left(S^{*}\right)$ of $S^{*}$ in virtue of the semi-regularity of $U(S)$ and of the semi-nilpotency ${ }^{5}$ of $L(S)$ is also semi-regular. Hence by Lemma 4 the ring $S^{*}$ contains a subring $T$ so that $U\left(S^{*}\right) \supseteq T=U(T) \supset L(T)$ and so that $S^{*}$ contains an infinite number of ideals $A_{1}^{*}, A_{2}^{*}, \cdots$ between $U(T)$ and $L(T)$ which are not radical ideals. Now define a subring $S^{\prime}$ of $S$ so that $S^{\prime} \supseteq L(S)$ and $S^{\prime} / L(S) \cong T$. By Lemma 3 it follows then that $S^{\prime}$ has the required properties.

THEOREM 6. If $S$ is a ring with a semi-nilpotent upper radical $U(S)$, and if $U(S) \supset L(S)$, then $S$ contains an infinite number of ideals between $U(S)$ and $L(S)$ which are not radical ideals.

Proof. Since by Lemma 3 the lower radical of the ring $S^{*}=S / L(S)$ is zero, it follows by Lemma 5 that the nonzero semi-nilpotent ideal $U(S) / L(S)$ of $S^{*}$ contains an infinite number of ideals which are not radical ideals. Our theorem follows now as a consequence of Lemma 3.

\section{BIBLIOGRAPHY}

1. R. Baer, Radical ideals, Amer. J. Math. vol. 65 (1943) pp. 537-568.

2. G. Koethe, Die Struktur der Ringe deren Restklassenring nach dem Radical vollstaendig reduzibel ist, Math. Zeit. vol. 32 (1930) pp. 161-186.

3. J. Levitzki, On the radical of a general ring, Bull. Amer. Math. Soc. vol. 49 (1943) pp. 462-466.

4. B. L. van der Waerden, Moderne Algebra, vol. 1, Berlin, 1937.

HEBREW UNIVERSITY 